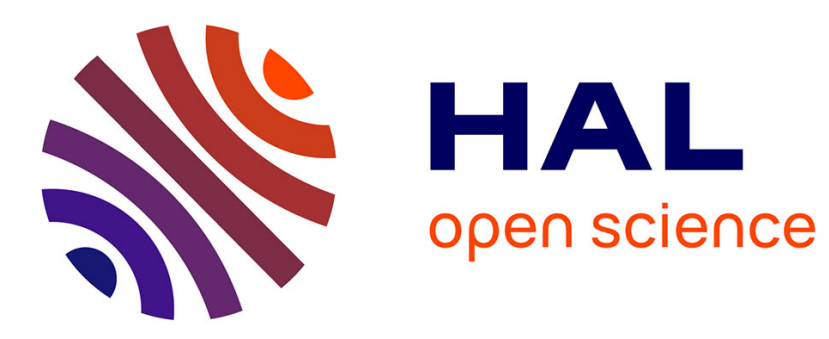

\title{
Démonstration du théorème d'Hermann à partir de la méthode Forte-Vianello
}

\author{
Nicolas Auffray
}

\section{To cite this version:}

Nicolas Auffray. Démonstration du théorème d'Hermann à partir de la méthode Forte-Vianello.

Comptes Rendus Mécanique, 2008, 336 (5), pp.458-463. 10.1016/j.crme.2008.02.003 . hal-00529633

\section{HAL Id: hal-00529633 \\ https://hal.science/hal-00529633}

Submitted on 26 Oct 2010

HAL is a multi-disciplinary open access archive for the deposit and dissemination of scientific research documents, whether they are published or not. The documents may come from teaching and research institutions in France or abroad, or from public or private research centers.
L'archive ouverte pluridisciplinaire HAL, est destinée au dépôt et à la diffusion de documents scientifiques de niveau recherche, publiés ou non, émanant des établissements d'enseignement et de recherche français ou étrangers, des laboratoires publics ou privés. 


\title{
Démonstration du théorème d'Hermann à partir de la méthode Forte-Vianello
}

\author{
Nicolas Auffray a \\ a ONERA-DMMP BP72 - 29 avenue de la Division Leclerc FR-92322 CHATILLON CEDEX
}

\section{Résumé}

A new derivation of Hermann'theorem is proposed, this demonstration is based on Forte-Vianello approach [1]. To cite this article: Auffray, C. R. Mécanique (200X).

\section{Résumé}

Une nouvelle dérivation du théorème d'Hermann est proposée. La démonstration s'appuiera sur les outils introduit par Forte et Vianello [1] . Pour citer cet article : Auffray, C. R. Mécanique (200X).

Key words: Tensor Algebra; Anisotropic Class

Mots-clés : Algèbre tensorielle; Classe d'anisotropie

\section{Introduction}

Un des théorèmes de C. Hermann [2] établit un lien formel entre l'ordre de symétrie d'un milieu matériel et celui d'une propriété tensorielle définie sur ce milieu. Ce théorème établit le fait que lorsque l'ordre d'un axe de symétrie excède l'ordre du tenseur de la propriété, alors celui-ci est le conjugué d'une classe de symétrie continue. Ce théorème issue de la cristallographie offre des perspectives très intéressantes vis-à-vis de la conception de matériaux architecturés. L'objectif de cette contribution est de présenter une nouvelle dérivation de ce résultat et ceci en insistant sur les mécanismes d'algèbres tensorielles sousjacents. Les outils utilisés pour ce faire sont issus de la théorie des groupes et sont en cohérence avec ceux introduits par Forte et Vianello en 1996 [1] lors de l'analyse des classes de symétrie de l'élasticité conventionnelle.

Email address: nicolas.auffray@onera.fr (Nicolas Auffray ). 


\section{Présentation des objets}

On considère un matériau $\mathcal{M}$ quelconque dont la matière occupe un domaine matériel $\mathcal{D}$ et nous appellerons $\mathcal{R}$ l'ensemble des variables caractérisant l'état de notre matériaux. Nous considérerons $\mathcal{A}$ l'environnement général de $\mathcal{M}$ (température, champs mécaniques, champs magnétiques,...). Nous appellerons propriété physique d'un matériau l'opérateur $\mathcal{F}$ qui traduit la manière dont notre matériau va s'adapter à une variation de son environnement, c'est-à-dire reliant une variation de l'environnement de notre matériau à une variation de son état. D'après [3] ceci peut s'écrire $\mathcal{F}: \mathcal{A} \longmapsto \mathcal{R}=\mathcal{F}\{\mathcal{A}\}$

Nous nous intéresserons à la restriction suivante du problème énoncé :

(i) nous considérerons le cas où $\mathcal{A}$ et $\mathcal{R}$ peuvent être représentés par des tenseurs cartésiens d'ordre $p$ en dimension 3. Nous considérerons de fait $\mathbb{T}^{(p)}$ l'espace vectoriel de ces tenseurs;

(ii) nous considérerons également que $\mathcal{F}$ est un automorphisme de $\mathbb{T}^{(p)}$, et nous noterons $\operatorname{GL}\left(\mathbb{T}^{(p)}\right)$ l'espace vectoriel associé. Nous avons de fait $\operatorname{GL}\left(\mathbb{T}^{(p)}\right) \subset \mathbb{T}^{(2 p)}$.

Introduisons maintenant les groupes de symétrie de notre domaine matériel ainsi que de notre propriété physique. Nous introduirons ensuite la notion de conjugaison qui nous permettra de comparer et de trier des objets abstraits entre eux.

\subsection{Symétries matérielles et physiques}

On considère $\mathrm{O}(3)$ le groupe orthogonal de $\mathrm{E}$, l'espace euclidien physique. Les symétries matérielles sont définies par l'invariance de $\mathcal{D}$, le domaine matériel, sous l'action de $\mathrm{G}_{\mathrm{m}}$, où $\mathrm{G}_{\mathrm{m}}$ est un sous-groupe fermé de $\mathrm{O}(3)$ défini de la manière suivante $: \mathrm{G}_{\mathrm{m}}=\{\mathrm{Q} \in \mathrm{O}(3) \mid \mathrm{Q} \star \mathcal{D}=\mathcal{D}\}$ où l'opérateur $\star$ représente le produit de Rayleigh.

On définit $\mathrm{G}_{\mathrm{p}}\left(\mathrm{T}^{(2 p)}\right)$ le groupe de symétrie du tenseur $\mathrm{T}^{(2 p)}$ :

$$
\mathrm{G}_{\mathrm{p}}\left(\mathrm{T}^{(2 p)}\right)=\left\{\mathrm{Q} \in \mathrm{O}(3) \mid \mathrm{Q} \star \mathrm{T}^{(2 p)}=\mathrm{T}^{(2 p)}\right\}, \mathrm{T}^{(2 p)} \in \mathrm{GL}\left(\mathbb{T}^{(p)}\right)
$$

Notre tenseur $\mathrm{T}^{(2 p)}$ qui traduit les propriétés physiques de notre matériau est d'ordre pair. L'écriture du produit de Rayleigh appliqué à un tenseur d'ordre pair fait apparaître un nombre pair de fois l'opérateur Q, de fait $G_{p}$ ne peut pas contenir l'inversion et on restreint $\mathrm{G}_{\mathrm{p}}\left(\mathrm{T}^{(2 p)}\right)$ à $\mathrm{SO}(3)$, le groupe spécial orthogonal. L'application du principe de Neumann [3] nous permet ensuite de relier ces deux groupes de symétries, i.e. $\mathrm{G}_{\mathrm{m}} \subseteq \mathrm{G}_{\mathrm{p}}$

\subsection{Relations de conjugaison}

Les notions ci-dessus permettent de caractériser un objet vis-à-vis de ses symétries. Pour comparer deux objets différents entre eux de manière à savoir s'ils sont, ou non, équivalents au sens de leurs symétries, on introduit la notion de groupes conjugués. Soient $\mathrm{G}_{1}$ et $\mathrm{G}_{2}$ deux sous-groupes conjugués de $\mathrm{SO}(3)$ on a :

$$
\mathrm{G}_{1} \sim \mathrm{G}_{2} \Leftrightarrow \exists \mathrm{R} \in \mathrm{SO}(3), \mathrm{G}_{1}=\mathrm{RG}_{2} \mathrm{R}^{T}
$$

La classe d'équivalence $\left\{G_{\alpha}\right\}$ d'un groupe $G_{\alpha}$ de $S O(3)$ est l'ensemble des sous-groupes qui lui sont conjugués, c'est-à-dire :

$$
\left\{\mathrm{G}_{\alpha}\right\}=\left\{\mathrm{G} \in \mathrm{SO}(3) \mid \exists \mathrm{R} \in \mathrm{SO}(3), \mathrm{G}_{\alpha}=\mathrm{RGR}^{T}\right\}
$$

On peut alors, à la manière de [1], définir des classes d'équivalence sur l'espace GL $\left(\mathbb{T}^{(p)}\right)$. On en retient la définition suivante :

$$
\mathrm{T}_{1}^{(2 p)}, \mathrm{T}_{2}^{(2 p)} \in\left(\mathrm{GL}\left(\mathbb{T}^{(p)}\right)\right)^{2}, \mathrm{~T}_{1}^{(2 p)} \sim \mathrm{T}_{2}^{(2 p)} \Leftrightarrow \exists \mathrm{R} \in \mathrm{SO}(3), \mathrm{G}_{p}\left(\mathrm{~T}_{1}^{(2 p)}\right)=\mathrm{RG}_{p}\left(\mathrm{~T}_{2}^{(2 p)}\right) \mathrm{R}^{T}
$$


Comme montré par Zheng et Boehler dans [3] seule la conjugaison vis-à-vis des sous-groupes fermés de $\mathrm{SO}(3)$ est à considérer. La collection de ces sous-groupes est $\Sigma:=\left\{I, Z_{m}, D_{m}, \mathcal{T}, \mathcal{O}, \mathcal{I}, \mathrm{SO}(2), \mathrm{O}(2), \mathrm{SO}(3)\right\}$, $(m \geq 2)$ où $:-I$ est le groupe identité; $Z_{m}$ le groupe cyclique d'ordre $m ; D_{m}$ le groupe diédral d'ordre $2 m ;-\mathcal{T}$ le groupe de symétrie du tétraèdre ; $\mathcal{O}$ le groupe de symétrie du cube ; - $\mathcal{I}$ le groupe de symétrie de l'icosaèdre. Le corollaire est que tout groupe de symétrie d'un tenseur est nécessairement le conjugué d'un unique élément de $\Sigma$ :

$$
\forall \mathrm{T}^{(2 p)} \in \mathrm{GL}\left(\mathbb{T}^{(p)}\right), \mathrm{G}_{\mathrm{p}}\left(\mathrm{T}^{(2 p)}\right) \sim \mathrm{G}_{\Sigma}, \mathrm{G}_{\Sigma} \in \Sigma
$$

L'espace vectoriel GL $\left(\mathbb{T}^{(p)}\right)$ est donc divisé en classes d'équivalences. Ces classes se définissent

$$
\mathrm{GL}\left(\mathbb{T}^{(p)}\right)\left(\mathrm{G}_{\Sigma}\right):=\left\{\mathrm{T}^{(2 p)} \in \mathrm{GL}\left(\mathbb{T}^{(p)}\right) \mid \mathrm{G}_{\mathrm{p}}\left(\mathrm{T}^{(2 p)}\right) \sim\left\{\mathrm{G}_{\Sigma}\right\}\right\}
$$

On notera que GL $\left(\mathbb{T}^{(p)}\right)\left(\mathrm{G}_{\Sigma}\right)$ ne constitue pas un sous-espace vectoriel de GL $\left(\mathbb{T}^{(p)}\right)$ ainsi que le fait que les classes d'équivalences sont disjointes, i.e.

$$
\mathrm{G}_{\Sigma_{1}} \neq \mathrm{G}_{\Sigma_{2}} \Rightarrow \mathrm{GL}\left(\mathbb{T}^{(p)}\right)\left(\mathrm{G}_{\Sigma_{1}}\right) \cap \mathrm{GL}\left(\mathbb{T}^{(p)}\right)\left(\mathrm{G}_{\Sigma_{2}}\right)=\varnothing
$$

On a de fait GL $\left(\mathbb{T}^{(p)}\right)=\bigcup_{\mathrm{G}_{\Sigma} \in \Sigma} \mathrm{GL}\left(\mathbb{T}^{(p)}\right)\left(\mathrm{G}_{\Sigma}\right)$. C'est à partir de ce matériel que nous allons dériver nos théorèmes.

\section{Théorèmes}

Nous pouvons résumer nos résultats au travers de 4 théorèmes :

Theorem 3.1 Soit $\mathrm{T}^{(2 p)}$ un tenseur cartésien d'ordre $2 p$ défini sur un domaine $\mathcal{D}$. Si le groupe de symétrie de $\mathcal{D}$ contient $Z_{m}$ avec $m>2 p$ alors le tenseur $\mathrm{T}^{(2 p)}$ est au moins hémitrope transverse.

Theorem 3.2 Soit $\mathrm{T}^{(2 p)}$ un tenseur cartésien d'ordre $2 p$ défini sur un domaine $\mathcal{D}$. Si le groupe de symétrie de $\mathcal{D}$ contient $D_{m}$ avec $m>2 p$ alors le tenseur $\mathrm{T}^{(2 p)}$ est au moins isotrope transverse.

Theorem 3.3 Soit $\mathrm{T}^{(2 p)}$ un tenseur cartésien d'ordre $2 p$ défini sur un domaine $\mathcal{D}$. Si le groupe de symétrie de $\mathcal{D}$ contient $Z_{m}$ avec $m>2 p$ et si la décomposition harmonique de $\mathrm{T}^{(2 p)}$ ne contient que des termes d'ordres pairs alors le tenseur $\mathrm{T}^{(2 p)}$ est au moins isotrope transverse.

De plus comme montré dans [4] la décomposition harmonique d'un tenseur totalement symétrique ne contient que des tenseurs d'ordres pairs de fait :

Theorem 3.4 Soit $\mathrm{T}^{(2 p)}$ un tenseur cartésien totalement symétrique d'ordre $2 p$ défini sur un domaine $\mathcal{D}$. Si le groupe de symétrie de $\mathcal{D}$ contient $Z_{m}$ avec $m>2 p$ alors le tenseur $\mathrm{T}^{(2 p)}$ est isotrope transverse.

\section{Démonstration}

Pour démontrer ces théorèmes nous nous baserons sur deux décompositions successives de notre tenseur d'ordre $2 p$. La première décomposition est une décomposition harmonique [5], la seconde une décomposition de Cartan [1]. Ces décompositions sont respectivement $\mathrm{SO}(3)$ et $\mathrm{SO}(2)$-invariante et nous permettent d'étudier facilement l'action d'un axe d'ordre $m$ sur un tenseur d'ordre $n$.

\subsection{Décomposition harmonique}

Tout tenseur cartésien peut être décomposé en une série de tenseurs harmoniques [5] d'ordres décroissants encapsulés dans des tenseurs d'ordres $k$ : 


$$
\mathrm{T}^{(n)}=\sum_{\oplus \tau, i} \mathrm{H}^{(i, \tau)}
$$

cette forme constitue le spectre de réduction de $\mathrm{T}^{(n)}, i$ indique l'ordre $\mathrm{du}$ tenseur harmonique, et $\tau$ distingue les différentes composantes de même ordre. Cette décomposition étant SO(3)-invariante [1] nous pouvons écrire :

$$
\forall \mathrm{Q} \in \mathrm{SO}(3), \forall \mathrm{T}^{(n)} \in \mathbb{T}^{(n)} \quad \mathrm{Q} \star \mathrm{T}^{(n)}=\left(\mathrm{Q} \star \mathrm{H}^{(n)}, \mathrm{Q} \star \mathrm{H}^{(n-1, \tau)}, \ldots, \mathrm{Q} \star \mathrm{H}_{l}^{(n-i, \tau)}, \ldots\right)
$$

Cette propriété nous permet d'écrire (1) le groupe de symétrie physique comme :

$$
\mathrm{G}_{\mathrm{p}}\left(\mathrm{T}^{(2 p)}\right)=\bigcap \mathrm{G}_{\mathrm{p}}\left(\{\mathrm{H}\}_{m}\right)
$$

avec $\{\mathrm{H}\}_{m}$ la collection de tous les tenseurs appartenant à la décomposition harmonique de $\mathrm{T}^{(2 p)}$. L'écriture du groupe de symétrie d'un tenseur comme intersection des groupes de symétrie des éléments de sa décomposition nous permet d'étudier chacun d'entre eux indépendamment.

\subsection{Décomposition de Cartan}

L'espace $\mathcal{H}^{q}$ des polynômes harmoniques de degré $q$ étant isomorphe à l'espace des tenseurs harmoniques d'ordre $q$, nous étudierons les polynômes associés à cette décomposition. Ces polynômes seront étudiés dans leur base de Cartan $\left\{\mathcal{K}_{i}^{q}\right\}_{1 \leq i \leq q}$ respective. L'invariance de $\mathrm{T}^{(2 p)}$ par l'action de $\mathrm{Q} \in \mathrm{G}_{\mathrm{m}}(\mathcal{D})$ se traduit par celle de ses composantes harmoniques. On considère le tenseur $\mathrm{H}^{(q)}$ issue de cette décomposition ${ }^{1}$ et d'ordre $q$. Son polynôme $h^{(q)} \in \mathcal{H}^{q}$, de degré $q$ s'exprime dans sa base de Cartan associée :

$$
h^{(q)}:=\lambda u+\sum_{i=1}^{q}\left(\alpha_{i} s_{i}+\beta_{i} t_{i}\right)
$$

Considérons à présent $h_{i}^{(q)}$ la composante de $h^{(q)}$ selon $\mathcal{K}_{i}^{q}$ et de degré $i: h_{i}^{(q)}=\alpha_{i} s_{i}+\beta_{i} t_{i}$. L'invariance de $\mathrm{T}^{(2 p)}$ par Q implique l'invariance de chaque composante de $h$ dans les sous-espaces de Cartan, c'est-à-dire

$$
\mathrm{Q} \star h_{i}^{(q)}=h_{i}^{(q)}
$$

Si l'on considère $\mathrm{Q}(\mathbf{k}, \theta) \in \mathrm{SO}(2)$ et $\mathrm{Q}(\mathbf{i}, \pi)$ leurs actions sur $\mathcal{K}_{i}^{q}$ s'écrivent respectivement :

$$
\left(\begin{array}{cc}
\cos (i \theta) & \sin (i \theta) \\
-\sin (i \theta) & \cos (i \theta)
\end{array}\right), \quad\left(\begin{array}{cc}
(-1)^{q-i} & 0 \\
0 & (-1)^{q-i+1}
\end{array}\right)
$$

On est ainsi passé d'une condition à vérifier sur l'ensemble du tenseur à une série de conditions matricielles portant sur les composantes des décompositions.

\subsection{Condition d'invariance}

D'après les relations précédentes, la condition (12) s'écrit :

$$
\alpha_{i}(\cos (i \theta)-1)-\beta_{i} \sin (i \theta)=0 \quad \text { et } \quad \alpha_{i} \sin (i \theta)-\beta_{i}(\cos (i \theta)-1)=0
$$

Ce système est vérifié

$$
\forall\left(\alpha_{i}, \beta_{i}\right) \in \mathbb{R}^{2} \operatorname{si} \theta=\frac{2 k \pi}{i}, \quad k \in \mathbb{N} \quad \text { ou } \quad \forall \theta \in[0,2 \pi] \text { si } \alpha_{i}=\beta_{i}=0
$$

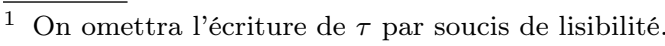


Action de $Z_{m}$ Si on considère l'invariance de $h^{(q)}$ par $\mathrm{Q}\left(\mathbf{k}, \frac{2 \pi}{m}\right)$ avec $m>q$, on a

$$
\forall i \in[1, q] \quad i \neq k m
$$

on ne peut donc satisfaire la condition $\operatorname{sur} \theta$ dans aucun des sous-espaces $\mathcal{K}_{i}^{q}$ avec $i \in[1, q]$; les coefficients associés a ces sous-espaces sont donc nuls. Il ne reste que le terme selon $\mathcal{K}_{0}^{q}$, soit :

$$
h^{(q)}:=\lambda u
$$

On a donc, pour un polynôme homogène harmonique de degré $q$, la propriété suivante :

$$
\forall \mathrm{Q} \in Z_{m}, m>\mathrm{d}^{\circ}\left(h^{(q)}\right) \quad \mathrm{Q} \star h^{(q)}=h^{(q)} \Leftrightarrow h^{(q)}=\lambda u
$$

par continuité si l'on considère que $\mathrm{SO}(2)=\lim _{m \rightarrow \infty} Z_{m}$ on peut récrire la propriété précédente :

$$
\forall \mathrm{Q} \in \mathrm{SO}(2) \quad \mathrm{Q} \star h^{(q)}=h^{(q)} \Leftrightarrow h^{(q)}=\lambda u
$$

De fait, vis-à-vis d'un polynôme homogène harmonique de degré $q$, une condition de symétrie d'ordre $q+1$ est équivalente à une condition de symétrie infinie. Par isomorphisme des espaces considérés cette propriété est transportable dans l'espace des tenseurs harmoniques d'ordre $q$. On abouti alors à

$$
\forall \mathrm{Q} \in Z_{m}, m>\mathcal{O}\left(\mathrm{H}^{(q)}\right) \Leftrightarrow\left(\forall \mathrm{Q} \in \mathrm{SO}(2) \quad \mathrm{Q} \star \mathrm{H}^{(q)}=\mathrm{H}^{(q)} \Leftrightarrow \mathrm{H}^{(q)}=\mathrm{H}_{0}^{(q)}\right)
$$

où $\mathrm{H}_{0}^{(q)}$ est le tenseur d'ordre 0 de la décomposition de Cartan du tenseur harmonique $\mathrm{H}^{(q)}$. L'application d'une condition de symétrie d'ordre $q+1$ sur un tenseur harmonique d'ordre $q$, est équivalente à l'application d'une condition de symétrie infinie. D'après la relation (19) ceci peut se récrire

$$
\forall \mathrm{Q} \in Z_{m}, m>\mathcal{O}\left(\mathrm{H}^{(q)}\right) \quad \mathrm{G}_{\mathrm{p}}\left(\mathrm{H}^{(q)}\right)=\mathrm{SO}(2)
$$

La composante harmonique d'ordre maximale de $\mathrm{T}^{(2 p)}$ étant d'ordre $2 p$, il suffit de choisir $m>2 p$ pour que l'on obtienne :

$$
\forall \mathrm{Q} \in Z_{m}, \mathrm{Q} \star \mathrm{T}^{(2 p)}=\mathrm{T}^{(2 p)} \Rightarrow \mathrm{G}_{\mathrm{p}}\left(\mathrm{T}^{(2 p)}\right)=\bigcap \mathrm{G}_{\mathrm{p}}\left(\{\mathrm{H}\}_{m}\right)=\mathrm{SO}(2)
$$

ce qui revient à dire que notre tenseur $\mathrm{T}$ est hémitrope transverse. Ceci prouve le théorème 3.1 .

Action de $D_{m}$ Considérons à présent l'action du groupe diédral $D_{m}$. Son action est équivalente à celle d'un groupe cyclique d'ordre $m$ auquel on rajouterait la rotation $Q(i, \pi)$ au générateur. Sous l'action de $Z_{m}$, avec $m>2 p$, la décomposition harmonique d'un tenseur d'ordre $2 p$ se réduit à l'ensemble des tenseurs $\mathrm{H}_{0}^{(q)}(20)$, ensemble qui est laissé invariant par $Z_{m}$. Si $\mathrm{Q}(\mathbf{i}, \pi)$ appartient au groupe de symétrie de ce tenseur, alors celui-ci vérifie :

$$
\mathrm{Q}(\mathbf{i}, \pi) \star \mathrm{T}^{(2 p)}=\mathrm{T}^{(2 p)}
$$

c'est à dire,

qui est vérifié

$$
\forall q \in[0,2 p] \quad \mathrm{Q}(\mathbf{i}, \pi) \star u=(-1)^{q} u=u
$$

$$
\forall u \text { si } q=2 t \quad \text { ou } \quad u=0 \text { si } q=2 t+1
$$

De fait si le groupe de symétries de $\mathcal{D}$ contient $D_{m}$ avec $m>2 p$ alors tous les termes impairs de la décomposition harmonique s'annulent et seuls subsistent ceux invariant par $Z_{2 p+1}$ et $\mathrm{Q}(\mathbf{i}, \pi)$, on a donc nécessairement pour $m>2 p$ :

$$
\forall \mathrm{Q} \in D_{m}, \mathrm{Q} \star \mathrm{T}^{(2 p)}=\mathrm{T}^{(2 p)} \Rightarrow \mathrm{G}_{\mathrm{p}}\left(\mathrm{T}^{(2 p)}\right)=\bigcap \mathrm{G}_{\mathrm{p}}\left(\{\mathrm{H}\}_{m}\right)=\mathrm{O}(2)
$$

ce qui revient à dire que notre tenseur $\mathrm{T}$ est isotrope transverse. Ceci prouve le théorème 3.2 . 
Pour les deux autres théorèmes il suffit de remarquer qu'il ne sert à rien d'éliminer les composantes impaires de la décomposition harmoniques si celles-ci n'existent pas. L'application stricte de cela aboutit au théorème 3.3 , tandis que le 3.4 note que cela est automatiquement vrai si l'on a affaire à un tenseur totalement symétrique.

\section{Synthèse et applications}

L'intérêt de ces théorèmes réside dans le fait qu'ils nous permettent d'obtenir très rapidement des informations sur une propriété physique quelconque liée à un milieu dont on connaît les symétries. Regardons quelques conséquences rapides que l'on peut tirer de ces théorèmes.

Élasticité classique Le spectre de réduction du tenseur d'élasticité, de part la présence de la grande symétrie, ne contient pas de termes d'ordre impair. On sait immédiatement que la classe de d'hémitropie transverse n'existe pas pour ce tenseur physique [1]. Si on supprime la grande symétrie, on retrouve le tenseur de l'effet Kerr, des termes d'ordre impair existent dans son spectre, on retrouve bien la classe d'hémitropie transverse [6]. De plus si l'on considère un milieu ayant une symétrie d'ordre 6 , alors comme l'ordre de la symétrie est supérieur à celui du tenseur d'élasticité, alors le milieu est isotrope transverse vis-à-vis de cette propriété.

Élasticité du second gradient Si l'on considère l'élasticité du second-gradient [7], on a la relation linéaire suivante entre le gradient de la déformation $\mathrm{K}^{(3)}$, le tenseur d'hypercontrainte $\mathrm{S}^{(3)}$ et le tenseur d'élasticité généralisée $\mathrm{A}^{(6)}: S_{i j k}=A_{i j k l m n} K_{l m n}$. Si l'on considère, à nouveau, un milieu ayant une symétrie d'ordre 6 , alors comme cette fois-ci l'ordre de la symétrie est égal à celui du tenseur d'élasticité du second ordre alors ce milieu isotrope transverse au premier ordre devient anisotrope à l'ordre deux. Comme de plus tout milieu périodique à une symétrie d'ordre inférieur ou égal à 6 , alors vis-à-vis de l'élasticité du second gradient tout milieu périodique est anisotrope.

Cette démonstration a été effectuée pour des raisons de concisions dans le cas spécial des tenseurs de comportement d'ordre pair. Sa généralisation à un tenseur d'ordre quelconque est directe.

\section{Références}

[1] S.Forte et M.Vianello. Symmetry classes for elasticity tensors, Journal of Elasticity 43 (1996) 81-108.

[2] C. Hermann. Tensoren und kristallsymmetrie. Zs. Kristallogr 8 (1934).

[3] Q.-S. Zheng et J. P. Boehler. The description, classification, and reality of material and physical symmetries. Acta Mechanica 102 (1994) 73-89.

[4] J. Jerphagnon, D. Chemla, et R. Bonneville. The description of the physical properties of condensed matter using irreducible tensors, Advances in Physics 27 (1978) 609-650.

[5] A. Spencer. A note on the decomposition of tensors into traceless symmetric tensors. International Journal of Engineering Science 8 (1970) 475-481.

[6] S.Forte et M.Vianello. Symmetry classes and harmonic decomposition for photoelasticity tensors. International Journal of Engineering Science 35 (1997) 1317-1326.

[7] Jean-Marc Cardona. Comportement et durée de vie des pièces multiperforées : application aux aubes de turbine. Thèse de doctorat, École Nationale Supérieure des Mines de Paris, 2000. 